\title{
Cosmological Evolution of the Rolling Tachyon
}

\author{
G W Gibbons \\ D.A.M.T.P., \\ C.M.S., \\ Cambridge University, \\ Wilberforce Road, \\ Cambridge CB3 0WA, \\ U.K.
}

November 26, 2017

\begin{abstract}
The cosmological effects of the tachyon rolling down to its ground state are discussed by coupling a simple effective field theory for the tachyon field to Einstein gravity. As the tachyon rolls down to the minimum of its potential the universe expands. Depending upon initial conditions, the scale factor may or may not start off accelerating, but ultimately it ceases to do so and the final flat spacetime is either static in the rest frame of the tachyon (if $k=0$ ) or ( if $k=-1$ ) given by the Milne model.
\end{abstract}




\section{Introduction}

The reconciliation of fundamental theories such as M/String theory with the basic facts of cosmology continues to present great challenges. The most straightforward approach is to pass to an effective field theory in which gravity is coupled to matter, for instance in a supergravity theory. Because the theories are formulated in higher dimensions, one must either construct a spontaneous compactification scenario or imagine a scheme in which the universe is sort of 3 -brane [3]. It seems to be very difficult to construct models with $N=1$ super-

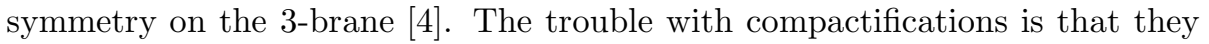
come with associated massless scalars and degenerate vacua: one must address the question of how these evolve with time [1]. One must also ensure that the resultant time-variability of coupling constants is compatible with observations. One possibility is to give the potentials a mass "by hand" such a way that Minkowski-space times a Calabi-Yau is an attractor at late times, [2] but this is ad hoc and ugly.

There is fairly good evidence from the BOOMERANG observations of the Cosmic Microwave Background that the scale factor of the universe underwent a period of acceleration (so-called Primordial Inflation) at early times and from Type Ia super-novae that it may also have been accelerating very recently if not today. (For a recent review see [8]). It is quite difficult to get accelerating universe out of pure supergravity theories 19, 10, 12, 13, 14, 11] although with super-matter, providing one gauges a suitable axial current this is possible [5]. The problem is that the axial gauging gives rise to anomalies [6]. It is possible that these anomalies can be cancelled in staring models with D-branes [7].

In recent years there has been great progress, particularly due to Sen, in our understanding of the role of the tachyon in String Theory (see 15 for a recent account with references to earlier work). The basic idea is that the usual open string vacuum is unstable but there exist a stable vacuum with zero energy density which is stable. There is evidence that this state is associated with the condensation of electric flux tubes of closed strings (see 15, 16]). These flux tubes described successfully using an effective Born-Infeld action (see [17, 15, 18] and references therein). This success of effective action methods, together with the difficulties of other approaches described the encourages one to pursue this further and to attempt a description of the cosmology of tachyon rolling. Moreover not to take into account the effects gravity during the process is inconsistent, since it involves a spatially uniform distribution of energy. It is

the purpose of this note to rectify this omission and initiate a study of tachyon cosmology .

\section{The Rolling Tachyon}

The tachyon of string theory may described by effective field theory describing some sort of tachyon condensate which in flat space has a Lagrangian density

$$
\mathcal{L}=-V(T) \sqrt{1+\eta^{\mu \nu} \partial_{\mu} T \partial_{\nu} T}
$$


where $T$ is the tachyon field, $V(T)$ is the tachyon potential and $\eta_{\mu \nu}=\operatorname{diag}(-,+,+, \ldots)$ is the metric of Minkowski spacetime ( see [16] for a discussion with references to earlier work). The tachyon potential $V(\bar{T})$ has a positive maximum at the origin and has a minimum at $T=T_{0}$ where the potential vanishes. In [16], $T_{0}$ is taken to lie at infinity. In Minkowski spacetime the rolling down of the towards its minimum value is described by a spatially homogeneous but time-dependent solution obtained from the Lagrangian density

$$
\mathcal{L}=-V \sqrt{1-\dot{T}^{2}} .
$$

During rolling the Hamiltonian density

$$
\mathcal{H}=\frac{V(T)}{\sqrt{1-\dot{T}^{2}}}
$$

has a constant value $E$. Thus

$$
\dot{T}=\sqrt{1-\frac{V^{2}(T)}{E^{2}}} .
$$

As $T$ increases $V(T)$ decreases and $\dot{T}$ increases to attain its maximum value of 1 in infinite time as $T$ tends to infinity. Note that as explained in 16 the tachyon field behaves like a fluid of positive energy density

$$
\rho=\frac{V(T)}{\sqrt{1-\dot{T}^{2}}}
$$

and negative pressure

$$
P=-V(T) \sqrt{1-\dot{T}^{2}}
$$

Thus

$$
P \rho=-V^{2}(T)
$$

and

$$
\frac{P}{\rho}=w=-\left(1-\dot{T}^{2}\right),
$$

and therefore, $-1 \leq w \leq 0$. Note that both the Weak Energy Condition, $\rho>0$ and Dominant Energy Condition, $\rho \geq|P|$ hold. However because

$$
\rho+3 P=-\frac{2 V(T)}{\sqrt{1-\dot{T}^{2}}}\left(1-\frac{3}{2} \dot{T}^{2}\right)
$$

the Strong Energy Condition fails to hold for small $|\dot{T}|$ but does hold for large $|\dot{T}|$.

The discussion above has neglected the gravitational field generated by the tachyon condensate. To take it into account we use the Lagrangian density

$$
\sqrt{-g}\left(\frac{R}{16 \pi G}-V(T) \sqrt{1-g^{\mu \nu} \partial_{\mu} T \partial_{\nu} T}\right),
$$


where $g_{\mu \nu}$ is the metric and $R$ its scalar curvature. We shall work in $3+$ 1 spacetime dimensions and assume that the metric has Friedman-LemaitreRobertson-Walker form

$$
d s^{2}=-d t^{2}+a^{2}(t) d \Omega_{k}^{2},
$$

where $a(t)$ is the scale factor and $d \Omega_{k}^{2}$ is, locally at least, the metric on $S^{3}, \mathbb{E}^{3}$ or $H^{3}$ according as $k=1,0,-1$ respectively. Note that in this model we have assumed that the cosmological constant $\Lambda$ vanishes in the tachyon ground state. The expressions (5) and (6) for the density and pressure remain valid and thus the Friedman and Raychaudhuri equations governing the evolution of the scale factor are

$$
\frac{\dot{a}^{2}}{a^{2}}+\frac{k}{a^{2}}=\frac{8 \pi G}{3} \frac{V(T)}{\sqrt{1-\dot{T}^{2}}},
$$

and

$$
\frac{\ddot{a}}{a}=\frac{8 \pi G}{3} \frac{V(T)}{\sqrt{1-\dot{T}^{2}}}\left(1-\frac{3}{2} \dot{T}^{2}\right) .
$$

The Hamiltonian density of the tachyon field is no longer constant because the tachyon Lagrangian density is now explicitly time dependent. Equations (2) and (3) must be replaced by

$$
\mathcal{L}=-a^{3} V \sqrt{1-\dot{T}^{2}} .
$$

and

$$
\mathcal{H}=a^{3} \frac{V(T)}{\sqrt{1-\dot{T}^{2}}} .
$$

The equation

$$
\frac{d \mathcal{H}}{d t}=-\frac{\partial \mathcal{L}}{\partial t}
$$

is formally equivalent to the conservation of entropy of the fluid which reads

$$
\dot{\rho}=-\frac{\dot{a}}{a}(\rho+P) .
$$

Because

$$
\rho+P=\frac{\dot{T}^{2}}{\sqrt{1-\dot{T}^{2}}}
$$

we have

$$
\frac{d}{d t}\left(\frac{V}{\sqrt{1-\dot{T}^{2}}}\right)=-\frac{3 \dot{a}}{a}\left(\frac{V \dot{T}^{2}}{\sqrt{1-\dot{T}^{2}}}\right) .
$$

Thus the evolution equation (蛋) remains valid but the quantity $E$ is no longer constant but rather decreases in time.

The course of cosmic evolution is now rather clear from these equations. The tachyon field rolls down hill with an accelerated motion and the universe 
expands. It still follows from (4) that as $T$ increases $V(T)$ increases but since $E$ decreases, in principal $\dot{T}$ could decrease but in any case $\dot{T}$ remains positive and so $T$ increases monotonically to attain its maximum value of 1 . From the Friedman equation (12), it follows that if $k \leq 0$, then $\dot{a}$ will always be positive. This is because the Weak Energy Condition holds, $\rho>0$. From the Raychaudhuri equation (13) one deduces that initially if $|\dot{T}|<\frac{2}{3}$ the scale factor

initially accelerates, $\ddot{a}>0$ but eventually, once $\dot{T}$ exceeds $\sqrt{\frac{2}{3}}$ the acceleration will cease and deceleration will set in. If the universe is flat, i.e. if $k=0$, then ultimately the scale factor will halt $a(t) \rightarrow$ constant. If the universe has hyperbolic sections, that is if $k=-1$, then ultimately the scale factor increases linearly with time, $a \rightarrow t$. In both cases the final state of the universe is flat, the case $k=-1$ being the Milne model. In the case of spherical sections, $k=+1$ re-collapse will take place. The possibility of cosmic acceleration arises from the positive potential $V(T)$ and should be contrasted with the situation in pure supergravity theories for which the Strong Energy Condition holds and cosmic acceleration is not possible [9, 10], unless the internal space is non-compact [12, 13]. The inclusion of supermatter matter may allow acceleration [14].

Despite the violation of the Strong Energy condition one sees from the Friedman equation (12) that in the cases $k \leq 0$ the positivity of energy precludes the avoidance of singularities in the past. If however $k=1$, it is conceivable that, for special initial conditions, the scale factor might pass through a finite sequence of minima and maxima or even that periodic or quasi-periodic solutions exist with an infinite sequence of maxima and minima.

\section{Acknowlegement}

After submitting this paper to the archive I was told of an earlier paper of Stephon Alexander [19] of which I was unaware and which anticipated some of the ideas discussed here, in terms of $D-\bar{D}$ annihilation. The action adopted for the tachyon field is different from that used in this paper. Another relevant pre-cursor brought to my attention by Anupam Mazumdar and to which the same remarks apply is 20]. I would like to thank them and also Andrew Chamblin, Neil Lambert, Mohammad Garousi, Thanu Padmanabhan, Nakoi Saskura and Arkady Tseytlin for helpful comments and pointng out typos and small inaccuracies in the wording.

\section{References}

[1] G W Gibbons and P K Townsend, Cosmological Evolution of Degenerate Vacua' Nucl Phys B282 (1987) 610

[2] G Chapline and G W Gibbons, Unification of Elementary Particle Physics and Cosmology in Ten dimensions Phys Letts B135(1984)43 
[3] G W Gibbons and D Wiltshire,'Spacetime as a Membrane in Higher Dimensions' Nucl Phys B 287(1987) 717, hep-th/0109093

[4] G W Gibbons and N D Lambert, 'Domain Walls and Solitons in Odd Dimensions' Phys Lett B488 (2000) 90-96, hep-th/0003197

[5] D Z Freedman, 'Supergravity with axial-gauge invariance' Phys Rev D15 (1977) 1173-1174

[6] D Z Freeman, private communication (December 2001)

[7] C Herdeiro, S Hirano and R Kallosh, String Theory and Hybrid Inflation/Acceleration JHEP 0102 (2001) 027, hep-th/0110271

[8] N Straumann, 'On the Cosmological Constant Problems and Astronomical Evidence for a Homogeneous Energy Density with Negative Pressure, astro-ph/020330

[9] G W Gibbons, 'Aspects of Supergravity Theories GIFT Seminar on Supersymmetry, Supergravity and Related Topics eds F. del Aguila, J A de Azcarraga and L E Ibanez, World Scientific(1984)

[10] J Maldacena and C Nunez,' Supergravity description of field theories on curved manifolds and a no go theorem' Int J Mod Phys A16 (2001) 822, hep-th/0007018

[11] S J Gates,' Is Stringy-Superysmmetry Quintessentially Challenged?', hep-th/0122112

[12] C M Hull, 'De-Sitter Space in Supergravity and M-Theory'JHEP 0109 (2001) 027, hep-th/0109213

[13] G W Gibbons and C M Hull, 'De-Sitter Space from Warped Supergravity Solutions', hep-th/0111072

[14] P K Townsend, 'Quintessence from M-Theory' JHEP 0111 (2001) 042, hep-th/0110072

[15] A Sen, 'Rolling Tachyon', hep-th/0203211

[16] A Sen, 'Tachyon Matter', hep-th/0203265

[17] G W Gibbons, K Hori and P Yi, ' String fluid from unstable D-branes, Nucl Phys B596 (2001) 136, hep-th/0009061

[18] A Sen, 'Fundamamental Strings in open string theory at the tachyonic vacuum' J Math Phys 42 (2001) 2844, hep-th/0010240

[19] S Alexander, 'Inflation from $D-\bar{D}$ Brane Annihilation, Phys Rev D65 (2002) 023507 hep-th/0105032

[20] A Mazumdar, S Panda and A Per/'ez-Lorenzan 'Assisted inflation via tachyon condensation' Nucl Phys B614 (2001) 101-116 hep-ph/0107058 